\title{
Do Transsacral-transiliac Screws Across Uninjured Sacroiliac Joints Affect Pain and Functional Outcomes in Trauma Patients?
}

\author{
John Heydemann MD, Braden Hartline MD, Mary Elizabeth Gibson MD, \\ Catherine G. Ambrose PhD, John W. Munz MD, Matthew Galpin RC, \\ Timothy S. Achor MD, Joshua L. Gary MD
}

Published online: 15 October 2015

(C) The Association of Bone and Joint Surgeons ( 2015

\begin{abstract}
Background Patients with pelvic ring displacement and instability can benefit from surgical reduction and instrumentation to stabilize the pelvis and improve functional outcomes. Current treatments include iliosacral screw or transsacral-transiliac screw, which provides greater biomechanical stability. However, controversy exists regarding the effects of placement of a screw across an uninjured sacroiliac joint for pelvis stabilization after trauma.

Questions/purposes Does transsacral-transiliac screw fixation of an uninjured sacroiliac joint increase pain and worsen functional outcomes at minimum 1-year followup compared with patients undergoing standard iliosacral

Each author certifies that he or she, or a member of his or her immediate family, has no funding or commercial associations (eg, consultancies, stock ownership, equity interest, patent/licensing arrangements, etc) that might pose a conflict of interest in connection with the submitted article.

All ICMJE Conflict of Interest Forms for authors and Clinical Orthopaedics and Related Research ${ }^{\circledR}$ editors and board members are on file with the publication and can be viewed on request.

Each author certifies that his or her institution approved the human protocol for this investigation, that all investigations were conducted in conformity with ethical principles of research, and that informed consent for participation in the study was obtained.
\end{abstract}

J. Heydemann, B. Hartline, M. E. Gibson, C. G. Ambrose, J. W. Munz, M. Galpin, T. S. Achor, J. L. Gary

Department of Orthopaedic Surgery, University of Texas

Medical School at Houston, Houston, TX, USA

\section{J. L. Gary $(\square)$}

Department of Orthopaedic Surgery, University of Texas

Medical School at Houston, 6400 Fannin Street, Suite 1700,

Houston, TX 77030, USA

e-mail: joshgary14@gmail.com screw fixation across the injured sacroiliac joint in patients who have sustained pelvic trauma?

Methods All patients between ages 18 and 84 years who sustained injuries to the pelvic ring (AO/OTA $61 \mathrm{~A}, \mathrm{~B}, \mathrm{C})$ who were surgically treated between 2011 and 2013 at an academic Level I trauma center were identified for selection. We included patients with unilateral sacroiliac disruption or sacral fractures treated with standard iliosacral screws across an injured hemipelvis and/or transsacraltransiliac screws placed in the posterior ring. Transsacraltransiliac screws were generally more likely to be used in patients with vertically unstable sacral injuries of the posterior ring as a result of previous reports of failures or in osteopenic patients. We excluded patients with bilateral posterior pelvic ring injuries, fixation with a device other than a screw, previous pelvic or acetabular fractures, associated acetabular fractures, and ankylosing spondylitis. Of the 110 patients who met study criteria, 53 (44\%) were available for followup at least 12 months postinjury. Sixty patients were unable to be contacted by phone or mail and seven declined to participate in the study. Outcomes were obtained by members of the research team using the visual analog scale (VAS) pain score for both posterior sacroiliac joints, Short Musculoskeletal Functional Assessment (SMFA), and Majeed scores. Patients completed the forms by themselves when able to return to the clinic. A phone interview was performed for others after they received the outcome forms by mail or email.

Results There were no differences between iliosacral and transsacral-transiliac in terms of VAS injured $(2.9 \pm 2.9$ versus $3.0 \pm 2.8$, mean difference $=0.1[95 \%$ confidence interval, -1.6 to 1.7$], \mathrm{p}=0.91)$, VAS uninjured $(1.8 \pm 2.4$ versus $2.0 \pm 2.6$, mean difference $=0.2$ [ -1.3 to 1.6$], \mathrm{p}=$ $0.82)$, Majeed $(80.3 \pm 19.9,79.3 \pm 17.5$, mean difference $=1.0[-11.6$ to 9.6], $\mathrm{p}=0.92)$, SMFA Function $(22.8 \pm$ 
22.2, $21.0 \pm 17.6$, mean difference $=1.8[-13.2$ to 9.6$], \mathrm{p}$ $=0.29$, and SMFA Bother $(24.3 \pm 23.8,29.7 \pm 23.4$, mean difference $=5.4[-7.8$ to 18.6$], \mathrm{p}=0.42$ ).

Conclusions Placement of fixation across a contralateral, uninjured sacroiliac joint resulted in no differences in pain and function when compared with standard iliosacral screw placement across an injured hemipelvis at least 1 year after instrumentation. When needed for biomechanical stability, transsacral-transiliac fixation across an uninjured sacroiliac joint can be used without expectation of positive or negative effects on pain or functional outcomes at minimum 1-year followup.

Level of Evidence Level III, therapeutic study.

\section{Introduction}

Iliosacral screw fixation of the posterior pelvic ring has become the standard treatment for unstable pelvic ring injuries as anatomical understanding of the osteology and its relation to fluoroscopic imaging has developed. Poorer outcomes have traditionally been associated with significant displacement and limb length inequality of the posterior ring and neurologic injury [2, 5]. These screws can be placed in a percutaneous fashion after open or closed reduction of the posterior ring $[1,3,4,8-12,14,18$, 19]. Screws can be placed into the sacral body (iliosacral screw) of first (S1) or second (S2) sacral segments. Screws may also be placed across the entire $\mathrm{S} 1$ or $\mathrm{S} 2$ segments in a transsacral-transiliac fashion if the patient's osteology allows. The proposed indications for transsacral-transiliac screws include: posterior pelvic instability, spinopelvic dissociation, osteoporosis, anticipated noncompliant behavior with unilateral screw fixation, bilateral posterior pelvic injuries, nonunions, and salvage procedures [1, 3, 18, 19]. Transsacral-transiliac screws anchor into iliac cortical bone on both sides of the pelvis, whereas standard iliosacral screws are placed in the less dense cancellous sacral ala [3]. Loss of reduction with iliosacral (IS) screw(s) and resultant malunion have been reported in $44 \%$ of vertically unstable ring injuries where only IS screws were used [3, 4, 6]. Transsacral-transiliac (TSTI) screws have provided a potential solution for these challenges because longer screws placed across six cortices gain better fixation than IS screws ending in the cancellous bone of the central sacrum, and TSTI screws create a longer level arm to resist vertical displacement for unilateral injuries. Biomechanical data support their improved fixation [18, 19].

The clinical effects of placing a screw across an uninjured joint, including potential increases in long-term sacroiliac joint pain and decreases in function, to improve biomechanical are unknown and further study have been suggested [3].

\section{Study Question}

Does TSTI screw fixation of an uninjured sacroiliac joint increase pain and worsen functional outcomes at 1-year followup compared with patients undergoing standard IS screw fixation across the injured hemipelvis in patients who have sustained pelvic trauma?

\section{Patients and Methods}

After obtaining institutional review board approval, our institutional orthopaedic trauma repository, which includes only operative injuries, at a university Level I trauma center was searched to identify all patients aged 18 to 84 years who sustained pelvic ring disruptions (AO/OTA 61 A, B, C) between September 1, 2011, and August 31, 2013, who had surgical stabilization $(n=222)$. Demographic and injury data for these patients were abstracted from the charts.

The 222 patients who underwent operative fixation for pelvic ring disruption were further categorized into groups of those with unilateral or bilateral sacroiliac disruption(s) and/or sacral fracture(s). Patients were excluded if they had bilateral posterior pelvic ring injuries, fixation with a device other than a screw, previous pelvic or acetabular fractures, associated acetabular fractures, and ankylosing spondylitis. The remaining 120 patients who underwent screw stabilization were then further divided into two groups: those with $(\mathrm{n}=53)$ and those without $(\mathrm{n}=87)$ TSTI screws. Early participating patients returned and participated voluntarily $(n=42)$. However, as a result of low followup rates, institutional review board approval was amended to provide patients a USD 50 financial incentive for travel and expenses. Eleven patients were enrolled after this incentive was offered. A total of 53 patients $(44 \%)$ returned to the clinic or completed outcomes measures by phone or mail at a minimum 12 months postoperatively. Average followup for our study group $(n=53)$ was 21.5 months (range, 12-33 months). Followup in the IS group (n $=31$ ) averaged 22.5 months (range, 12-31 months) and in the TSTI group $(n=22)$ averaged 20.0 months (range, 1233 months). There were no statistically significant differences in age, gender, OTA/AO classification, or Injury Severity Score (ISS) between patients treated with IS or TSTI fixation (Table 1). Multiple attempts were made to contact all other patients. Sixty were unable to be contacted and seven declined to participate in the study. Thirty-seven 
Table 1. Patient demographics

\begin{tabular}{llll}
\hline Variable & Iliosacral $(\mathrm{n}=31)$ & $\begin{array}{l}\text { Transsacral- } \\
\text { transiliac }(\mathrm{n}=22)\end{array}$ & $\mathrm{p}$ value \\
\hline Age (years; SD) & $40(10.3)$ & $42(16.2)$ & 0.672 \\
Gender & & & \\
Male & 17 & 15 & 0.4 \\
Female & 14 & 7 & \\
OTA/AO classification & & 0.946 \\
61-A & 0 & 0 & \\
61-B & 11 & 8 & 0.945 \\
61-C & 20 & 14 & \\
ISS (SD) & $23.4(10.3)$ & $23.6(10.5)$ &
\end{tabular}

ISS = Injury Severity Score.

Table 2. Patient demographics of eligible patients not enrolled

\begin{tabular}{llll}
\hline Variable & $\begin{array}{l}\text { Iliosacral } \\
(\mathrm{n}=37)\end{array}$ & $\begin{array}{l}\text { Transsacral- } \\
\text { transiliac } \\
(\mathrm{n}=30)\end{array}$ & $\begin{array}{l}\text { p value comparing } \\
\text { aggregate Table 1 } \\
\text { with aggregate } \\
\text { Table 2 }\end{array}$ \\
\hline $\begin{array}{l}\text { Age (years; SD) } \\
\text { Gender }\end{array}$ & $35(11.7)$ & $43(17.7)$ & 0.546 \\
Male & 21 & 21 & 0.851 \\
Female & 16 & 9 & \\
OTA/AO classification & & \\
61-A & 1 & 1 & 0.356 \\
61-B & 17 & 9 & \\
61-C & 19 & 20 & 0.322 \\
ISS (SD) & $21.5(9.8)$ & $22.5(9.9)$ &
\end{tabular}

ISS = Injury Severity Score.

patients (54.4\%) in the IS group and 30 patients $(58.8 \%)$ in the TSTI group were excluded for insufficient followup. In the group of eligible patients who were not enrolled $(\mathrm{n}=$ 67), there were no statistically significant differences in age, gender, OTA/AO classification, or ISS when the group excluded as a result of insufficient followup was compared with the study groups (Table 2).

There was no formal protocol for use of TSTI fixation and method of fixation was chosen at the discretion of the fellowship-trained orthopaedic trauma surgeon based on the fracture pattern, bone quality, and individual patient osteology of the posterior pelvic ring. TSTI screws were generally more likely to be used in patients with vertically unstable injuries of the posterior ring as a result of previous reports of failures [4] or in osteopenic patients; however, the decision to use IS or TSTI fixation was made according to the judgment of the attending traumatologist. The techniques used were described in the technique paper by Gardner and Routt [3]. Two-dimensional fluoroscopy was used for all cases.
Postoperatively, all patients were mobilized with physical therapy. Touchdown weightbearing was generally prescribed for the injured posterior hemipelvis and weightbearing as tolerated was allowed contralaterally for 8 to 12 weeks unless other lower extremity injuries precluded this. Patients were followed by their respective surgeons in the clinic and were contacted to return at 1 year from the surgery. All patients were made weightbearing as tolerated for the bilateral lower extremities by the time of followup for the study; however, only one was not ambulating as a result of a cerebrovascular accident, which occurred during the initial injury rendering the patient confined to a wheelchair.

For the patients who were able to return to the clinic, AP, inlet, and outlet radiographs of the pelvis were obtained at no financial cost to the patient to assess for reduction and healing.

Outcomes were assessed using the visual analog scale (VAS) pain score for both posterior sacroiliac joints (the injured and uninjured sides), Short Musculoskeletal Functional Assessment (SMFA), and Majeed scores. The Majeed score includes five factors of pain, sitting, standing, sexual intercourse, and work performance [7]. The SMFA scoring system evaluates patients through a two-part, 46item self-reported health status examination [13]. Patients completed the forms themselves when able to return to the clinic. If unable to return to the clinic, a phone interview was performed by a member of the research team other than the attending surgeon after they received the outcome forms by mail or email.

\section{Statistical Analysis}

Mean values for each group were compared using a standard two-tailed t-test. The significance of difference between the two groups was calculated using a two-tailed Fisher's exact test. Level of statistical significance was defined as $\mathrm{p}<0.05$.

\section{Results}

There were no differences between IS and TSTI in terms of VAS injured $(2.9 \pm 2.9$ versus $3.0 \pm 2.8$, mean difference $=0.1$ [95\% confidence interval, -1.6 to 1.7$], \mathrm{p}=0.91$ ), VAS uninjured $(1.8 \pm 2.4$ versus $2.0 \pm 2.6$, mean difference $=0.2[-1.3$ to 1.6$], p=0.82)$, Majeed $(80.3 \pm 19.9$, $79.3 \pm 17.5$, mean difference $=1.0[-11.6$ to 9.6$], \mathrm{p}=$ 0.92), SMFA Function $(22.8 \pm 22.2,21.0 \pm 17.6$, mean difference $=1.8[-13.2$ to 9.6], $\mathrm{p}=0.29$, and SMFA Bother $(24.3 \pm 23.8,29.7 \pm 23.4$, mean difference $=5.4$ [ -7.8 to 18.6$], \mathrm{p}=0.42$ ) (Table 3$)$. 
Table 3. Functional outcomes between the IS and TSTI groups enrolled in the study

\begin{tabular}{|c|c|c|c|c|}
\hline \multirow[t]{2}{*}{ Parameter } & $\begin{array}{l}\text { Iliosacral } \\
(\mathrm{n}=31)\end{array}$ & $\begin{array}{l}\text { Transsacral-transiliac } \\
(\mathrm{n}=22)\end{array}$ & Mean difference $(95 \% \mathrm{CI})$ & $\mathrm{p}$ value \\
\hline & Mean (SD) & Mean (SD) & & \\
\hline VAS Injured SI & $2.87(2.9)$ & $2.95(2.8)$ & $0.08(-1.6$ to 1.7$)$ & 0.912 \\
\hline VAS Uninjured SI & $1.84(2.4)$ & $2.00(2.6)$ & $0.16(-1.3$ to 1.6$)$ & 0.819 \\
\hline Majeed & $80.3(19.9)$ & $79.3(17.5)$ & $1.00(-11.6$ to 9.6$)$ & 0.917 \\
\hline SMFA Function & $22.8(22.2)$ & $21.0(17.6)$ & $1.80(-13.2$ to 9.6$)$ & 0.286 \\
\hline SMFA Bother & $24.3(23.8)$ & $29.7(23.4)$ & $5.40(-7.8$ to 18.6$)$ & 0.415 \\
\hline
\end{tabular}

IS = iliosacral; TSTI = transacral-transiliac; CI = confidence interval; VAS = visual analog score; SI = sacroiliac; SMFA = Short Musculoskeletal Functional Assessment.

We performed a post hoc power calculation using a beta of 0.2 (corresponding to $80 \%$ power) and holding alpha at 0.05 or less; were the small observed differences in VAS scores to hold up, a sample size of 13,000 patients per group would be needed to show a significant difference in VAS scores, and, again, that difference would still not be clinically important.

\section{Discussion}

Outcomes research after pelvic ring injury remains a limited field. Little is known regarding the long-term effects of malreduction or transarticular fixation through the IS joint. Retrospective data have shown that limb length inequality after pelvic fracture results in poorer outcomes [5]. Currently, there are no published comparisons of which we are aware between crossing and not crossing an uninjured sacroiliac joint in patients with pelvic trauma. We therefore sought to determine whether TSTI screw fixation of an uninjured sacroiliac joint worsens pain and increases functional outcomes at 1-year followup compared with patients undergoing standard IS screw fixation across the injured hemipelvis in patients who have sustained pelvic trauma. We found no significant differences in VAS pain scores of the injured or uninjured sacroiliac joint or SMFA function and bother indices between trauma patients who did or did not have TSTI fixation across an uninjured hemipelvis.

There are several limitations to this retrospective study. Our followup of $44 \%$ is a major limitation. Long-term followup in the trauma population is a well-known issue for outcomes research [17]. In general, the concern is that loss to followup in studies of newer treatments results in overestimation of the benefits of those newer treatments, because patients lost to followup may have undergone reoperations or revision surgery that are not captured by the analysis or have had complications treated elsewhere.
However, our study found no apparent benefits to placement of screws across uninjured sacroiliac joints; if some patients had problems or complications, that would have further depressed the pain and functional scores compared with what we observed. There was no differential loss to followup between our two groups and no differences were seen with regard to age, sex, fracture pattern, or ISS between our study group and those excluded as a result of lack of followup. Other limitations include lack of a formal protocol to indicate TSTI fixation and multiple surgeons using different techniques for screw placement. Although we generally choose TSTI fixation for vertically unstable injuries or osteopenic patients, the lack of prospective collection of reasoning of choice for IS versus TSTI fixation introduces room for selection bias. The finding of no difference in fracture classification between our groups ameliorates some of the concern. Multiple surgeons of varying levels of experience could also have a positive or negative impact on outcomes. However, all surgeons in our center use both IS and TSTI screws with similar indications for more robust fixation, and an advantage of multiple surgeons is that these results should be able to generalize to a broader surgeon population than a single-surgeon study. Routine screw removal may also change outcomes at 1 year [16]; we do not routinely perform this at our center and any affect is beyond the scope of this study. Finally, a possible explanation for the findings in any no-difference study is insufficient statistical power. We do not believe that was the explanation here, because the observed effect sizes were very small, some even smaller than the minimum clinically important differences for the outcomes tool in question such as was the case for VAS pain [15]. Based on our post hoc power calculation, for the small observed differences in VAS scores to hold up, a sample size of 13,000 patients per group would be needed to show a significant difference in VAS scores, and, again, that difference would still not be clinically important. 
Our data at 1 year suggest that crossing an uninjured sacroiliac joint with a TSTI screw at S1 and/or S2 has no effect, positive or negative, on patient pain or functional outcome. Zhao et al showed that TSTI provides superior biomechanical fixation than IS screws in finite element analysis and cadaveric models [18, 19]. This type of fixation has been proposed as a solution to previous failures of IS screws with vertically unstable sacral fractures according to Griffin et al [4]. A few small retrospective studies have shown good clinical results with TSTI screws [1, 3]. Gardner and Routt analyzed functional outcome assessments at least 1 year after surgery in 18 patients who underwent TSTI fixation; no comparison group was used [3]. Our SMFA bother and function indices for both the IS and TSTI screw groups are similar to those published by Gardner and Routt in an expert single-surgeon series, further supporting that our results can be generalized. To our knowledge, this study is the first to compare outcomes with and without fixation across an uninjured sacroiliac joint for unilateral injuries to the posterior ring.

We found no differences in VAS pain scores, Majeed scores, or SMFA scores at 1 year between patients with and without TSTI screws for unilateral injuries to the posterior pelvic ring. The indications for TSTI screw fixation should be evaluated carefully in future studies to determine which patients will benefit from them. Multicenter, prospective, observational studies of pelvic ring injuries would shed light on the need for, and type of, surgical fixation and the effect on long-term outcomes, if any. However, when needed for biomechanical stability, especially in pelvic ring injuries with vertical instability, we conclude that TSTI fixation across an uninjured sacroiliac joint can be used without expectation of positive or negative effects on pain or functional outcomes at minimum 1-year followup.

Acknowledgments We thank Cary Warner and Jeffery Tomasek MD, of the Center for Translational Research at The University of Texas Medical School at Houston for assistance in patient demographics. We also thank Michelle Kwan MD, for assistance in gathering patient demographics.

\section{References}

1. Beaule PE, Antoniades J, Matta JM. Trans-sacral fixation for failed posterior fixation of the pelvic ring. Arch Orthop Trauma Surg. 2006;126:49-52.

2. Denis F, Davis S, Comfort T. Sacral fractures: an important problem. Retrospective analysis of 236 cases. Clin Orthop Relat Res. 1988;227:67-81.
3. Gardner MJ, Routt ML Jr. Transiliac-transsacral screws for posterior pelvic stabilization. J Orthop Trauma. 2011;25:378-384.

4. Griffin DR, Starr AJ, Reinert CM, Jones AL, Whitlock S. Vertically unstable pelvic fractures fixed with percutaneous iliosacral screws: does posterior injury pattern predict fixation failure? $J$ Orthop Trauma. 2006;20:S30-36; discussion S36.

5. Henderson RC. The long-term results of nonoperatively treated major pelvic disruptions. J Orthop Trauma. 1989;3:41-47.

6. Keating JF, Werier J, Blachut P, Broekhuyse H, Meek RN, O'Brien PJ. Early fixation of the vertically unstable pelvis: the role of iliosacral screw fixation of the posterior lesion. J Orthop Trauma. 1999;13:107-113.

7. Majeed SA. Grading the outcome of pelvic fractures. $J$ Bone Joint Surg Br. 1989;71:304-306.

8. Matta JM, Tornetta P 3rd. Internal fixation of unstable pelvic ring injuries. Clin Orthop Relat Res. 1996;329:129-140.

9. Routt ML Jr, Simonian PT. Closed reduction and percutaneous skeletal fixation of sacral fractures. Clin Orthop Relat Res. 1996;329:121-128.

10. Routt ML Jr, Simonian PT, Mills WJ. Iliosacral screw fixation: early complications of the percutaneous technique. J Orthop Trauma. 1997;11:584-589.

11. Sagi HC, Militano U, Caron T, Lindvall E. A comprehensive analysis with minimum 1 -year follow-up of vertically unstable transforaminal sacral fractures treated with triangular osteosynthesis. J Orthop Trauma. 2009;23:313-319; discussion 319-321.

12. Schildhauer TA, Josten C, Muhr G. Triangular osteosynthesis of vertically unstable sacrum fractures: a new concept allowing early weight-bearing. J Orthop Trauma. 2006;20:S44-51.

13. Swiontkowski MF, Engelberg R, Martin DP, Agel J. Short musculoskeletal function assessment questionnaire: validity, reliability, and responsiveness. $J$ Bone Joint Surg Am. 1999;81:1245-1260.

14. Tabaie SA, Bledsoe JG, Moed BR. Biomechanical comparison of standard iliosacral screw fixation to transsacral locked screw fixation in a type C zone II pelvic fracture model. J Orthop Trauma. 2013;27:521-526.

15. Tashjian RZ, Deloach J, Porucznik CA, Powell AP. Minimal clinically important differences (MCID) and patient acceptable symptomatic state (PASS) for visual analog scales (VAS) measuring pain in patients treated for rotator cuff disease. $J$ Shoulder Elbow Surg. 2009;18:927-932.

16. Yucel N, Lefering R, Tjardes T, Korenkov M, Schierholz J, Tiling T, Bouillon B, Rixen D. [Is implant removal after percutaneous iliosacral screw fixation of unstable posterior pelvic ring disruptions indicated?] [in German]. Unfallchirurg. 2004;107: 468-474.

17. Zelle BA, Bhandari M, Sanchez AI, Probst C, Pape HC. Loss of follow-up in orthopaedic trauma: is $80 \%$ follow-up still acceptable? J Orthop Trauma. 2013;27:177-181.

18. Zhao Y, Li J, Wang D, Liu Y, Tan J, Zhang S. Comparison of stability of two kinds of sacro-iliac screws in the fixation of bilateral sacral fractures in a finite element model. Injury. 2012;43:490-494.

19. Zhao Y, Zhang S, Sun T, Wang D, Lian W, Tan J, Zou D, Zhao Y. Mechanical comparison between lengthened and short sacroiliac screws in sacral fracture fixation: a finite element analysis. Orthop Traumatol Surg Res. 2013;99:601-606. 